\title{
DIMENSIONES ANTROPOMÉTRICAS DE LA MANOY EL PIE EN EL RECIÉN NACIDO Y SU RELACIÓN CON EL PESO Y LA TALLA
}

\section{DIMENSIONS ANTROPOMETRICS HAND AND FOOT IN NEWBORN AND ITS RELATION TOTHE WEIGHT ANS SIZE}

\author{
Abelardo Rodríguez Menendez ${ }^{1}$
}

RESUMEN

\begin{abstract}
El conocimiento minucioso de las dimensiones antropométricas del recién nacido normal tiene importancia no sólo para discernir acerca que si cierto hallazgo debe ser o no considerado patológico, sino también porque puede constituir una vía para evaluar precozmente las potencialidades y aptitudes físicas internas del sujeto. Se estudiaron 341 recién nacidos, a quienes se les midió la longitud y ancho de la mano, ancho del pulgar, longitud del tercer dedo de la mano, longitud y ancho del pie, ancho del primer dedo del pie, peso y talla. Mediante un programa para tal efecto, se calcularon las medianas y los percentiles $3,10,90 \mathrm{y}$ 97. También se estimaron y compararon los valores medios de cada grupo por sexo y raza. Por último, se realizó un análisis de regresión múltiple de todas las variables con el peso y la longitud supina del niño. Los resultados indican diferencias atribuibles al sexo y la raza en varias mediciones. Igualmente encontramos correlaciones más altas con la longitud supina que con el peso, como cabía esperar. Es necesario continuar estos estudios a fin de su validación.
\end{abstract}

Palabras clave: Longitud de mano, longitud del tercer dedo, ancho de mano

\section{ABSTRACT}

\begin{abstract}
A minutely precise knowledge of anthropometric dimensiones of normal newborn is significant, not only to discrimínate if certain finding must be considered or not as pathologic, but also because it may be a way for an early evaluation of internal physical aptitudes and potencialities of the individual. Three hundred and forty one newborns, whose lenght and wide of the hand, wide of thumb, lenght of third finger, lenght and wide of the foot, wide of first toe, weight and helght, being measured, were studied. Through a programme builded up to such effect, medians and 3,10,90 and 97 percentils were calculated. Mean values of each group by sex and race were calculated and compared. Finally, a multiple regression analysis of all variables with weight and supine lenght of the child was performed. Results show differences imputable to sex and race in several measurements. Likely as it was supposed higher correlations to supine lenght than with weight, were found. To go on with these studies for their validation, is a need.
\end{abstract}

Keywords: Length of hand, third finger length, hand width

\section{I.-INTRODUCCIÓN}

$\mathrm{El}$ conocimiento minucioso de las dimensiones antropométricas del recién nacido ( $\mathrm{RN})$ normal tiene importancia para discernir acerca de si cierto hallazgo debe o no ser considerado patológico, porque puede constituir una vía para evaluar precozmente las potencialidades $y$ aptitudes físicas internas del sujeto.
En la época actual, el estudio de la anatomía y la antropometría ha estado dirigido hacia el desarrollo y el crecimiento del hombre y la valoración de cómo influyen ciertos factores nutricionales, en estos procesos. [1]-[5]

En el presente trabajo nos proponemos investigar si existen diferencias antropométricas de manos y pies entre los niños 
del grupo estudiado, y específicamente, si existen tales diferencias entre el niño recién nacido de raza negroide y europoide, así como determinar el grado de correlación entre las variables estudiadas en la mano y el pie, y con el peso y la talla.

\section{II.- MATERIALES Y MÉTODOS}

Para el presente trabajo se tomó una muestra de $34.1 \mathrm{RN}$, en el hospital materno "Eusebio Hernández", ciudad de La Habana-CUBA. En ellos se realizaron las siguientes mediciones:

- Peso del recién nacido (PRN)

- Longitud supina o talla(LS)

- Ancho de la mano(AM)

- Ancho del primer dedo de la mano(A1DM)

-Longitud de la mano(LM)

- Longitud del tercer dedo de la mano(L3DM)

- Ancho del pie (AP)

- Ancho del primer dedo del pie(A1DP)

-Largo del pie (8LP)

El peso se determinó en gramos y las mediciones de longitud en $\mathrm{mm}$. El análisis estadístico se realizó mediante un programa confeccionado. Se estimaron y compararon las medias de cada variable, se halló el coeficiente de correlación entre el peso y la talla, así como la de éstos con el resto de las variables estudiadas. Se compararon además, las medias de las variables por sexo y raza.

\section{III.- RESULTADOS}

La media $(\times)$, desviación estándar $(\mathrm{S})$, valor mínimo (V. min.), valor máximo (V.máx.) y error estándar (ES) de las variables estudiadas se muestran en la Tabla I.

Los coeficientes de correlación entre las variables estudiadas se muestran en la Tabla II, donde observamos que el mayor coeficiente se presenta entre el peso y la longitud supina, y en todos existe una significación entre el ancho del primer dedo de la mano y la longitud de su tercer dedo. Por otra parte, hallamos la ecuación de regresión para el peso del $\mathrm{RN}$ (Tabla III). Hemos eliminado la variable longitud supina, debido a que conocemos que el coeficiente de correlación entre peso y longitud supina es muy elevado.

TABLA I

DESCRIPCIÓN ESTADÍSTICA GENERAL ( $\mathrm{N}=34.1)$

\begin{tabular}{|l|r|r|r|r|r|r|r|r|r|}
\cline { 2 - 11 } \multicolumn{1}{c|}{} & 1 & \multicolumn{1}{c|}{2} & \multicolumn{1}{c|}{3} & \multicolumn{1}{c|}{4} & \multicolumn{1}{c|}{5} & \multicolumn{1}{c|}{6} & \multicolumn{1}{c|}{8} & \multicolumn{1}{c|}{9} \\
\cline { 2 - 11 } \multicolumn{1}{c|}{} & PRN & \multicolumn{1}{c|}{ LS } & AM & A1DM & LM & L3DM & AP & A1DP & \multicolumn{1}{c|}{ LP } \\
\hline X & 3250 & 503 & 32,9 & 8,28 & 62,5 & 27,8 & 31,3 & 9,89 & 74,8 \\
\hline S & 467 & 25,9 & 2,8 & 1,1 & 4,2 & 2,8 & 2,7 & 1,0 & 5,1 \\
\hline V. Mín. & 2029 & 384 & 24 & 5 & 52 & 18 & 25 & 7 & 57 \\
\hline V. Máx. & 4430 & 617 & 42 & 12 & 74 & 37 & 39 & 13 & 87 \\
\hline ES & 1,171 & 0,276 & 0,091 & 0,056 & 0,111 & 0,09 & 0,090 & 0,054 & 0,122 \\
\hline
\end{tabular}

TABLA II

COEFICIENTE DE CORRELACIÓN ENTRE LAS VARIABLES ESTUDIADAS

\begin{tabular}{|c|c|c|c|c|c|c|c|c|c|}
\cline { 2 - 10 } \multicolumn{1}{c|}{} & 1 & 2 & 3 & 4 & 5 & 6 & 7 & 8 & 9 \\
\cline { 2 - 10 } \multicolumn{1}{c|}{} & PRN & LS & AM & A1DM & LM & L3DM & AP & A1DP & LP \\
\hline 1 & - & 0,662 & 0,477 & 0,388 & 0,494 & 0,356 & 0,487 & 0,399 & 0,531 \\
\hline 2 & & - & 0,437 & 0,312 & 0,494 & 0,394 & 0,443 & 0,388 & 0,472 \\
\hline 3 & & & - & 0,430 & 0,397 & 0,323 & 0,574 & 0,481 & 0,533 \\
\hline 4 & & & & - & 0,270 & 0,104 & 0,364 & 0,409 & 0,288 \\
\hline 5 & & & & & - & 0,497 & 0,415 & 0,299 & 0,479 \\
\hline 6 & & & & & & - & 0,312 & 0,275 & 0,311 \\
\hline 7 & & & & & & & - & 0,486 & 0,459 \\
\hline 8 & & & & & & & & - & 0,343 \\
\hline 9 & & & & & & & & & - \\
\hline
\end{tabular}


TABLA III

ECUACIÓN DE REPRESIÓN PARA EL PESO DEL RECIÉN NACIDO

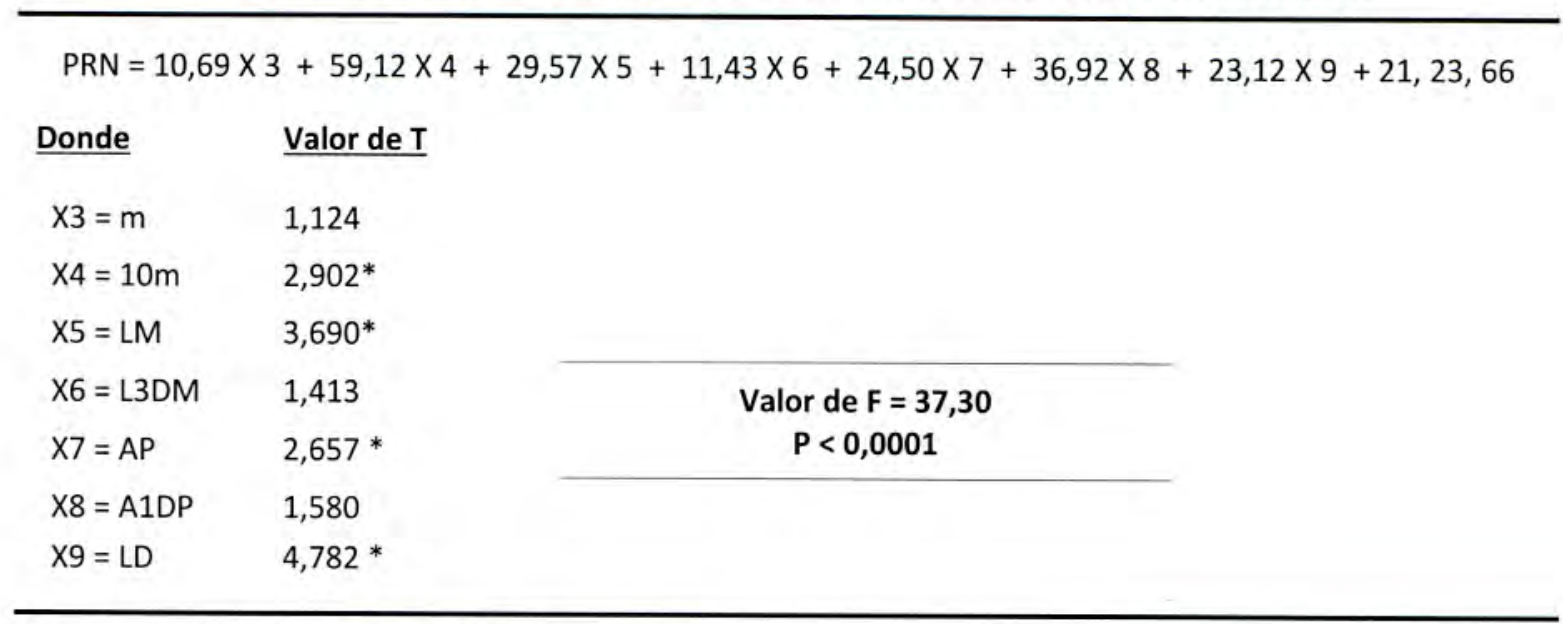

* Variables relevantes: $\mathrm{X} 9, \mathrm{X} 5, \mathrm{X} 4, \mathrm{X7}$

El valor de $\mathrm{F}$ en el estadígrafo utilizado es muy alto con $\mathrm{P}<0,0001$. Para los valores de $\mathrm{T}$ observados, las variables más relevantes son: X9, $\mathrm{X} 5, \mathrm{X}_{4}$ y X7, en sentido decreciente.

La ecuación de regresión para la longitud supina del $\mathrm{RN}$ se expresa en la tabla IV, donde observamos que el valor de $\mathrm{F}$ en el estadígrafo es de 30,13 ( $\mathrm{P}<0,0001)$; por lo que, esta ecuación se ajusta al valor real. Las variables más relevantes son X5, X9 y X6, en sentido decreciente.

La comparación de las medias de las variables estudiadas según el sexo muestra diferencias significativas en todos los casos, excepto en la longitud del tercer dedo de la mano (Tabla V).

El comportamiento de las variables estudiadas según los rasgos raciales predominantes en la madre aparece en la tabla VI.

De las 9 variables estudiadas, el peso del $\mathrm{RN}$ y la longitud supina mostraron ser significativamente mayores en los europoides $(\mathrm{P}<$ 0,05 y $\mathrm{P}<0,005$ respectivamente), mientras que la longitud de la mano y la longitud del tercer dedo de la mano fueron mayores en la raza negroide $(\mathrm{P}$ $<0,01$ y $\mathrm{P}<0,05$ respectivamente). No se encontraron diferencias significativas en las cinco restantes variables estudiadas.

TABLA IV

ECUACIÓN DE REPRESIÓN PARA LA LONGITUD DEL RECIÉN NACIDO

$$
\text { LS / 0,59X3 + 1,41X4 +1,35X5+1,19X6+1,07X7+2,89 X8+0,94X9+221,25 }
$$

$\begin{array}{lll}\text { Donde } & & \text { Valor de T } \\ \text { X3 }=\mathrm{m} & 1,077 \\ \mathrm{X} 4=10 \mathrm{~m} & 1,197 \\ \mathrm{X} 5=\mathrm{LM} & 3,993^{*} \\ \mathrm{X} 6=\text { L3DM } & 2,553 * \\ \text { X7 }=\text { AP } & 1,999 \\ \text { X8 }=\text { A1DP } & 2,140 \\ \text { X9 }=\text { LD } & 3,392\end{array}$

* Variables relevantes: X5, X9, X6 


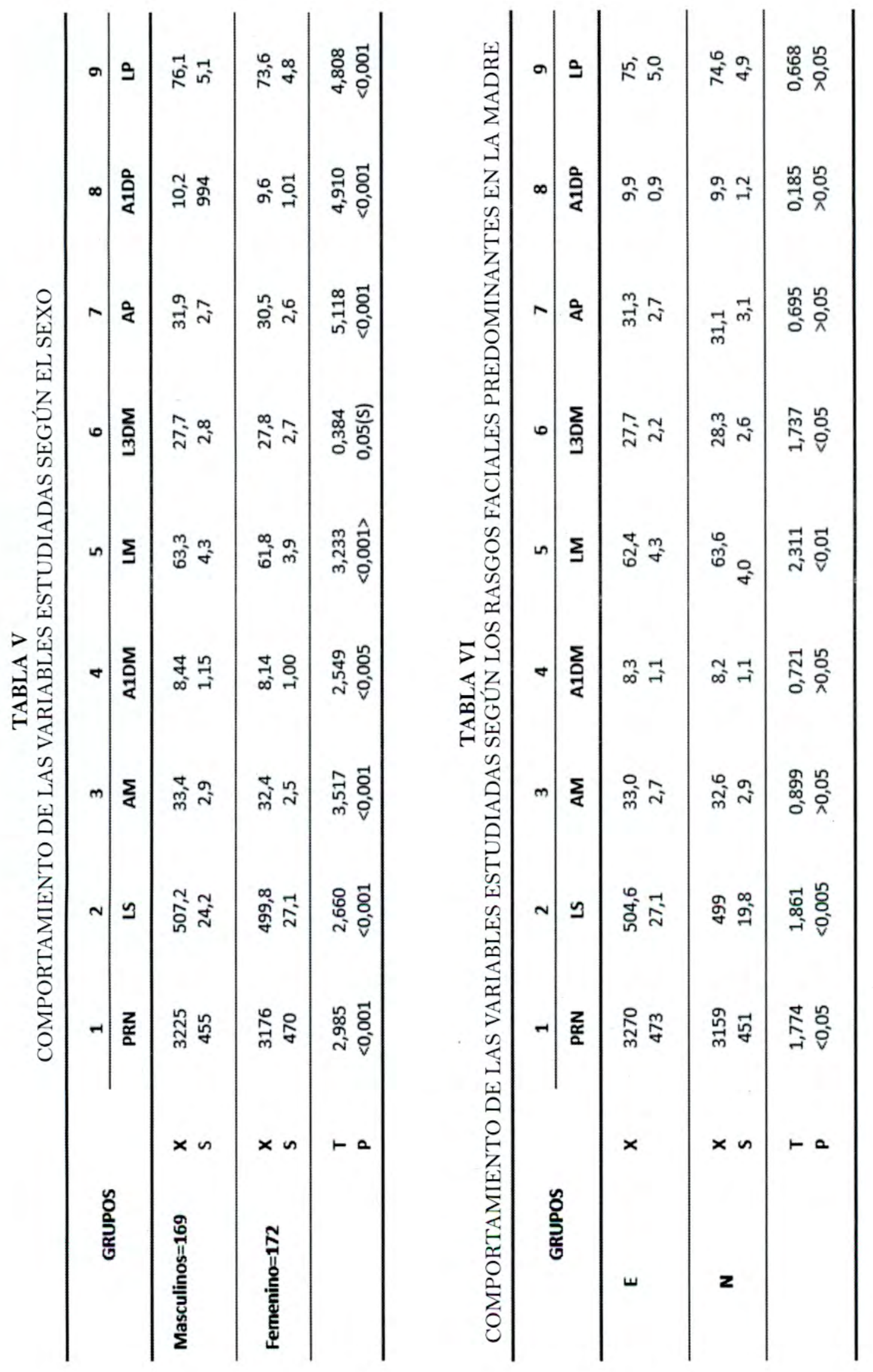




\section{IV.- CONCLUSIONES}

- Las variables de mano y pie estudiadas mostraron una correlación importante, excepto entre el ancho del primer dedo de la mano con el largo del tercer dedo de la mano.

- Los valores de correlación fueron más altos con la longitud que con el peso.

- En general, las variables estudiadas en pies y manos tuvieron valores significativamente mayores en varones que en hembras.

- Se encontraron valores mayores en el peso y la talla de los recién nacidos europoides, y en la longitud de la mano y la longitud del tercer dedo de la mano de los negroides. Mientras que no se encontraron diferencias significativas en el resto.

\section{REFERENCIAS BIBLIOGRÁFICAS}

[1] DUOST. Z.B : Dietary restriction and fetal development. Experientia 33: 1371, 1977.
[2] EDOZIEN, J. C: Influence of diet on growing in the rat. J. Nutr 108: 282, 1978.

[3] FELDEM, J.M. et al: Effect of food restriction on mice with hereditary obesity. J Nutr 126: 162, 1979

[4] FERNANDEZ, G. et al: Influence of diet on survival of mice. Proc Nati Acad Sci USA, 1279, 1979.

[5] LEDERMAN S.A.: Rosso: Effect of food restriction on fetal and placental growing and maternal bodu composition. Growth 44: 77, 1980.

\section{Correspondencia:}

Abelardo Rodríguez Menéndez

Ciudad Universitarias fundo "Los Granados"

Av. Miraflores s/n Tacna - Perú

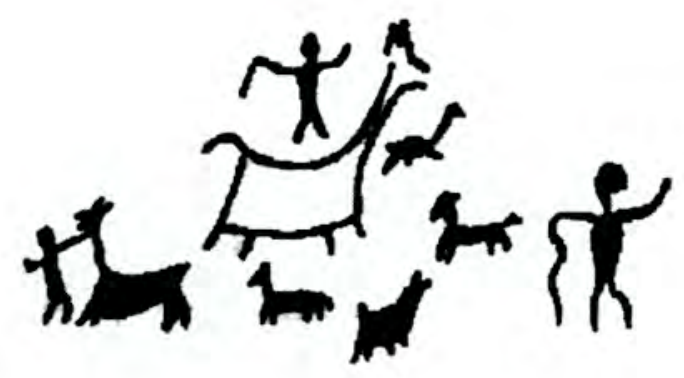

